\title{
TTR
}

Traduction, terminologie, re?daction

\section{Le pouvoir anonyme : les voix passives dans le procès de $\mathrm{K}$.}

\section{Éliane Morillon-Räkel}

Volume 5, numéro 2, 2e semestre 1992

Kafka pluriel : réécriture et traduction

URI : https://id.erudit.org/iderudit/037125ar

DOI : https://doi.org/10.7202/037125ar

Aller au sommaire du numéro

Éditeur(s)

Association canadienne de traductologie

ISSN

0835-8443 (imprimé)

1708-2188 (numérique)

Découvrir la revue

Citer cet article

Morillon-Räkel, É. (1992). Le pouvoir anonyme : les voix passives dans le procès de K. TTR, 5(2), 125-140. https://doi.org/10.7202/037125ar d'utilisation que vous pouvez consulter en ligne.

https://apropos.erudit.org/fr/usagers/politique-dutilisation/ 


\section{Le pouvoir anonyme: les voix passives dans le procès de $K$.}

\section{Éliane Morillon-Räkel}

Jemand mußte Josef $K$. verleumdet haben, denn ohne $\mathrm{daß}$ er etwas Böses getan hătte, wurde er eines Morgens verhaftet. (p. 7) ${ }^{1}$

Il fallait qu'on ait calomnié K.: un matin, sans avoir rien fait de mal, il fut arrêté. (Lortholary, p. 29) ${ }^{2}$

1. Franz Kafka, Der Prozess (Frankfurt, Fischer Bucherei, 1960).

2. Notre étude tiendra compte des trois traductions du Procès:

1) celle d'Alexandre Vialatte (Gallimard, 1933), citée d'après Franz Kafka, le Procès, traduit de l'allemand avec une introduction d'Alexandre Vialatte, préface de Bernard Groethuysen (Paris, Gallimard, coll. «Folio», 101, 1975);

2) celle de Bernard Lortholary, citée d'après Franz Kafka, le Procès, traduction et introduction de Bemard Lortholary (Paris, Flammarion, 1983);

3) celle de Georges-Arthur Goldschmidh, citée d'après Franz Kafka, le Procès, traduction nouvelle et présentation de Georges-Arthur Goldschmidt (Paris, Presses Pocket, 1983). 
Cette entrée en matière, la première phrase du roman le Procès, semble augurer d'un récit en bonne et due forme; pourtant, par son contenu et son style, elle contient déjà les signes des situations paradoxales et absurdes auxquelles sera confronté K.: «Er wurde eines Morgens verhaftets (un matin il fut arrêté). Excepté la date bien vague du moment de l'arrestation et l'hypothèse de la trahison, les circonstances et les participants resteront inconnus; le texte enchaîne en effet sur:

Die Köchin der Frau Grubach, seiner Zimmervermieterin, die ihm jeden Tag gegen acht Uhr früh das Frühstück brachte, kam diesmal nicht. (p. 7)

La cuisinière de Madame Grubach, sa logeuse, ne lui apporta pas son petit déjeuner, comme elle le faisait tous les jours vers huit heures. (Lortholary, p. 29),

il laisse donc le lecteur sur ses questions et la suite du récit de l'arrestation échappe à tout essai de reconstitution logique:

Gleichzeitig befremdet und hungrig, läutete er. (p. 7)

Puis, intrigué en même temps qu'affamé, il sonna. (Lortholary, p. 29)

Dès lors, partagé entre son instinct de vie et le monde qui se dérobe, le sujet K. deviendra étranger à son environnement et à lui-même.

Ces premières lignes du roman le Procès mettent déjà en place les éléments narratifs et discursifs qui, par leur récurrence et leur effet d'insistance, vont marquer la trame du roman. Pour mettre en langue, en mots et en structure l'histoire de K., Kafka va chercher dans la langue allemande, langue avec laquelle il entretenait, semble-t-il, le rapport ambigu dont il a souvent été question ${ }^{3}$, les formulations qu'il côtoyait dans le monde de son travail. Employé aux services juridiques

Lorsque nous citerons une des traductions, nous indiquerons entre parenthèses le nom du traducteur.

3. Régine Robin, Kafka (Paris, Pierre Belfond, 1989). Cf. le chapitre «Kafka et la langue de l'Autre» (pp. 37 et suiv.). 
d'une compagnie d'assurances, il fréquente un allemand bureaucratique; c'est cet allemand minimal, formel, dépersonnalisé, vide de tout sentiment et apparemment objectif, l'allemand de la jurisprudence, truffé de formules toutes faites derrière lequel se retranche l'employé subalterne, celui-là même et celui-là seul qui apparaît à travers divers dédoublements tout au long du Procès. Ironiquement donc, Kafka emprunte à son quotidien ses pratiques discursives: parmi celles-ci le, les passifs. Ainsi K. dira:

Es wurde gar nichts untersucht, ich wurde nur verhaftet. ( $p$. 25)

Il n'y a eu d'enquête sur rien du tout, j'ai simplement été arrêté. (Goldschmidt, p. 57)

Forme composite, le passif allemand est une forme verbale dont le véritable pivot est l'auxiliaire 'werden': cet élément, déterminé par le lexème verbal, dont le sens premier est celui du devenir, exprime clairement qu'un processus évolutif est en cours; il décrit une dynamique: «ich wurde verhaftet». Le participe passé, quant à lui, renverse cette dynamique et envisage le processus rétrospectivement, lui donne une marque finie, irrévocable, un effet de non-retour. Dynamisme et immobilisme coexistent donc dans cette forme de passif. Cette structure qui est omniprésente dans la narration du Procès - à l'exception de quelques rares passages où $\mathrm{K}$. assume entièrement le déroulement de l'action - nous présente le personnage de $\mathrm{K}$. dans toute son ambiguitté. Personnage qui s'engage de plus en plus et sacrifie ses intérêts professionnels au profit de sa cause, K. est aussi le spectateur d'une dynamique dont il est de moins en moins maître puisque sa fin est paradoxalement déjà inscrite, programmée, pré-déterminée. De surcroit, cette forme grammaticale est avant tout et presque essentiellement une forme verbale pouvant se passer de référents; les exécutants de l'action (aides, subalternes, avocat malade, peintres, femme, fille, ceux que K. appelle «niedrige Organe»), ces éternels intermédiaires qui ne sont en fin de compte que des figurants sont radiés du récit. Ainsi dans le chapitre intitulé Advokat - Fabrikant Maler (l'avocat, l'industriel, le peintre) et qui servira désormais à notre analyse, sur 70 formes passives, 7 sont, grammaticalement parlant, accompagnées de leur complément d'agent. Bien plus encore, cette forme verbale peut même renoncer à son sujet grammatical. «Es wurde 
nichts untersucht»: le pronom neutre 'es' sans contenu précis et que l'on appellera 'sujet apparent', annonciateur du vrai sujet «nichts» peut même disparaître comme dans les exemples suivants:

wie offen zugestanden werden soll (p. 91)

il faut le dire franchement (Lortholary, p. 162)

ou encore:

wurde ihm entgegnet (p. 91)

on lui rétorquait (Lortholary, p. 163)

Le processus engagé peut même se passer du principal protagoniste:

als werde über seinem Kopf von zwei Männern (...) über ihn selbst verhandelt (p. 96)

$K$. eut l'impression de deux hommes (...) en train de décider de lui par-dessus sa tête (Goldschmidt, p. 158)

«Je ne pense ni à ceux qui y poussent, ni à ceux qui sont poussés. Je vois seulement ce qui se passe. Les personnages sont tout à fait accessoires», ${ }^{4}$ dira Kafka à son jeune ami Gustav Janouch en parlant du «déracinement». L'action échappe au personnage principal qui assiste à une transformation, à une métamorphose. Le sujet est mis en dehors, mis à l'écart, désintéressé. $\mathrm{K}$. assiste à la liquidation de sa personne et perd le contrôle de ses actes, ce qui lui inspire la remarque suivante:

es gehört zu der Art dieses Gerichtswesens, daß man nicht nur unschuldig, sondern auch unwissend verurteilt wird. (p. 40) c'est bien dans la manière de ces tribunaux de condamner des innocents qu'on laisse de surcroît dans l'ignorance. (Lortholary, pp. 91-92)

K. va même jusqu'à ignorer ses sensations:

4. Gustav Janouch, Conversations avec Kafka, traduit de l'allemand par Bemard Lortholary (Paris, Maurice Nadeau, 1978), p. 68. 
da er an die Hitze erinnert worden war... (p. 114)

maintenant qu'on venait de lui rappeler la chaleur... (Goldschmidt, p. 183)

La structure grammaticale du passif en 'werden' est donc l'élément discursif par excellence qui exprime à la fois le processus ainsi que l'incontournable, l'insaisissable, l'insondable de celui-ci. Le sujet-spectateur glisse ainsi doucement vers un état de fait qui s'exprime dans le texte par une structure grammaticale alternant avec la précédente, c'est le 'deuxième passif' que les grammairiens appelleront parfois 'passif-état'; cette forme verbale se distingue de la précédente par l'élément déterminé: c'est l'auxiliaire 'sein' qui, lui, est vide de tout contenu dynamique. Pour cette raison, ce passif est peut-être le seul des deux à mériter son nom. ${ }^{5}$ Ainsi, dès le premier chapitre, madame Grubach commente ce qui arrive à $K$. en ces termes:

5. J.M.Zemb dans Vergleichende Grammatik Französisch-Deutsch, Teil 1 (Mannheim, Duden-Verlag, 1978, p. 472) analyse le passif comme suit (je traduis en abrégeant): «Les représentations qu'évoque le terme "passif" et les concepts liés à ces représentations supposent un renversement du rapport sujet-objet; à la voix active, on entend la voix de l'agent, à la voix passive on entend la voix du patient.(...) Le renversement des rôles des foncteurs régis est en effet inévitable si et seulement si les foncteurs sont représentés. Cette conception-type du passif sous-entend la transitivité et les conversions qu'elle rend possibles. En considérant 'être battu' et 'getragen-werden' comme des formes de base de 'tragen', on s'est mépris sur la structure du taxème proprement dit. Le noyau de 'est battu', de 'gesagt wird', de 'geschrieben ist', voire de 'geschrieben steht', n'est pas P(articipe) mais V(erbe conjugué). Ce que l'on appelle "nouveau sujet" est gouvemé par (est une valence de) est, wird, ist, steht! Nous n'avons donc pas à faire à un nouveau sujet en face d'un verbe connu mais d'un nouveau verbe avec son sujet (s'il en a besoin). P est un attribut (dans certains cas cet attribut peut être thématisé), un déterminant à l'intérieur du thème. On avait dans le domaine de $\mathrm{P}$, considéré comme un lexème, un soi-disant "porteur de l'action". Ce dernier pourra, sous l'étiquette "complément d'agent", être considéré comme indication de la cause, de la condition, du moyen (introduite par 'de', 'par', 'von', 'durch')." 
Sie sind zwar verhaftet, aber nicht so wie ein Dieb verhaftet wird. Wenn man wie ein Dieb verhaftet wird, so ist es schlimm, aber diese Verhaftung -. (p. 20)

Vous êtes arrêté, c'est vrai, mais non pas comme est arrêté un voleur. Quand on est arrêté comme l'est un voleur, c'est grave. (Goldschmidt, p. 49)

'Diese Verhaftung' qui se lit parfois 'das Verhaftetsein', c'est l'arrestation en tant qu'état, état que $K$. atteint avant même que le processus ne soit expliqué. D'ailleurs, cet état n'est-il pas jugé comme une fin positive?

Es kommt mir wie etwas Gelehrtes vor. (p. 20)

Je trouve que ça a quelque chose de savant. (Lortholary, p. 47)

Ainsi s'exprime la logeuse, madame Grubach, faisant une distinction très nette entre l'acte d'arrestation et l'état d'arrestation, à moins que madame Grubach ne soit victime des apparences. «La détention - dira Kafka à $\mathrm{G}$. Janouch - est organisée comme une existence quotidienne tout à fait ordinaire, sans confort excessif. Tout semble construit dans un matériau solide et stable. Mais en fait c'est un ascenceur qui descend à toute allure vers l'abîme.» (lbid., p. 69) Le passage du processus à l'état ou plus exactement la différence fondamentale telle que la pressent la logeuse et telle qu'elle est transmise par le jeu des deux formes du passif marque la trame des différents récits qui composent le Procès. À titre d'exemple, on notera ainsi que le premier chapitre comporte 29 passifs exprimant un processus et 48 décrivant un état; le chapitre sept quant à lui compte 70 passifs du premier type (donc passif en 'werden'), 47 du second type (passif en 'sein'). De par son ambivalence, le passif allemand n'est-il pas l'expression de ce que Régine Robin appelle «figure de l'entre-deux, de la transformation constante (déplacement/condensation), de l'atermoiement illimité qui sont des signifiants clés chez Kafka»? (Ibid., p. 125) Le va-et-vient d'un passif à l'autre, du processus à l'être, à la soumisssion, à la résignation reflète le combat au prix duquel $\mathrm{K}$. semble acquérir une forme de liberté. La métamorphose de $\mathrm{K}$. passe ainsi par différents stades: «ich bin verhaftet» a été précédé de «ich wurde verhaftet» et de «ich bin verhaftet worden». Le résultat qui trouve son expression dans cette forme du passif dit passif-état prend alors une valeur de qualité 
positive et semble représenter une fin en soi; au début du récit, $\mathrm{K}$. s'adresse un reproche:

Man ist aber so wenig vorbereitet. (p. 20)

on est tellement peu préparé! (Goldschmidt, p. 50),

qu'il reprend plus loin comme un constat d'échec:

Als eine Grundregel für das Verhalten eines Angeklagten erschien es ihm, immer vorbereitet zu sein (...) - und gerade gegen diese Grundregel verstieß er immer wieder. (p. 121)

Être toujours préparé, ne jamais se laisser surprendre, (...) voilà qui lui avait toujours paru être la règle de base du comportement de l'accusé; or c'était à cette règle qu'il contrevenait toujours. (Goldschmidt, p. 192)

Au dernier chapitre, K. constatera au sujet des deux bourreaux:

Sie sind nicht darauf vorbereitet, gefragt zu werden. (p. 162) Ils n'ont pas l'habitude qu'on les interroge. (Goldschmidt, p. 252)

Traduire en français la dualité du personnage de $\mathrm{K}$. et des situations qu'il rencontre, traduire «l'entre-deux», transmettre le cheminement ou l'oscillation du processus vers l'état, voire une certaine qualité d'être, faire passer la quête d'une disponibilité, c'est traduire fidelement les structures grammaticales des deux passifs; or le passif français est une forme verbale ambiguê; le seul auxiliaire être dont il est formé ne permet pas de distinguer le devenir de l'état. Pour réaliser les deux contenus sémantiques présents dans les passifs allemands en 'werden' ou en 'sein', la forme passive française a, on le sait, besoin de conditions bien spécifiques. Ainsi, dans les énoncés 'K. fut arrêté', 'K. sera arrêté', 'K. est arrêté', c'est le seul changement de temps de l'auxiliaire du passif qui nous fait passer de la notion de processus à celle d'état. D'autres éléments parviennent à supprimer la polysémie de la forme verbale, tel le contenu inchoatif, voire imperfectif, du lexème verbal: dans les deux exemples 'la requête est transmise' et 'la requête est adoptée', l'aspect lexical subvient au manque de forme grammaticale 
univoque. Autre élément permettant de situer l'action dans sa dynamique ou à l'état fini, la mention de l'agent responsable de l'action: 'K. est arrêté par des gardes' décrit le processus, 'K. est arrêté' l'état final. Autre différence: contrairement à la forme allemande, le passif français ne saurait se passer de la mention du protagoniste et parfois même du responsable de l'action (soit du sujet grammatical et de l'agent). Forme verbale se suffisant à elle-même, le passif allemand en 'sein' figure un état, celui en 'werden' permet quant à lui d'entrevoir l'action pure et ceci à partir de verbes intransitifs aussi ('es wurde gelacht': 'lachen'/'rire' étant un verbe sans complément); cette possibilité est évidemment exclue en français. Il apparaît que, selon la langue considérée, les contenus du passif ne peuvent être transmis de la même façon. En allemand, ces contenus résultent automatiquement de l'auxiliaire déterminé par le participe; en français, ils sont transmis par des éléments soit morphologiques, soit lexicaux.

À la lumière d'une comparaison des trois traductions, nous nous proposons de montrer jusqu'où vont les limites de l'adéquation de ces formes auxquelles les grammairiens donnent une étiquette identique. Nous ferons porter notre comparaison sur le chapitre déjà mentionné et intitulé: Advokat - Fabrikant - Maler (l'avocat, l'industriel, le peintre). C'est le récit de trois rencontres au cours desquelles $\mathrm{K}$. se fait expliquer les différentes procédures possibles et commence à prendre part à son procès. Sa participation se trouve pourtant neutralisée, voire objectivée par la perspective que donnent les formes verbales. Procès, cause, requête, recours y apparaissent comme des processus déshumanisés et prédéterminés:

Der Prozess muß eben immerfort in dem kleinen Kreis, auf den er künstlich eingeschränkt worden ist, gedreht werden. ( $p$. 118)

Au sujet des requêtes, on lit:

daß die ersten Eingaben bei Gericht gar nicht gelesen würden (p. 85)

Puis il est fait allusion à certaines situations, 
wo keine Hilfe mehr geleistet werden darf (p. 91)

Puis le rôle passif du sujet est décrit comme suit:

Alles muß auf den Angeklagten selbst gestellt sein. (p. 86)

Ces quelques exemples montrent combien dans ce chapitre l'approche par le et les passifs est cruciale.

Si nous faisons une analyse cas par cas des trois traductions du Proces, force nous est de constater que très peu de formes passives trouvent leur équivalent en français. Ainsi, sur 70 passifs en 'werden', sur lesquels nous nous attarderons en premier, les trois traductions ne présentent que cinq, quinze, vingt calques parfaits des formes allemandes - parfait voulant dire qu'il s'agit de la structure passive telle qu'elle apparaît en français avec l'auxiliaire 'être' et le participe passé. Bien que ces trois traductions présentent des pourcentages différents (nous reviendrons sur cette différence), le nombre de transpositions littérales reste malgré tout faible. Ces transpositions remplissent les conditions énumérées ci-dessus: la présence du complément d'agent, le temps de l'auxiliaire, le contexte ou le contenu sémantique du lexème verbal lèvent l'ambiguité de la forme polysémique. Dans la traduction de «daß die ersten Eingaben... gar nicht gelesen würden» (p. 85) par «que les premières requêtes (...) n'y soient lues par personne» (Lortholary, p. 154), c'est le complément d'agent qui permet de percevoir l'aspect dynamique; dans la traduction de «Der Direktor-Stellvertreter (...) wurde (...) dadurch nur aufgemuntert) (pp. 96-97) par «Mais le directeur adjoint (...) en fut tout au plus émoustillé» (Lortholary, p. 169), c'est le contenu non perfectif de la forme verbale. De même, le passage «Wenn ihnen ein Prozeß (...) plötzlich aus der Hand genommen wird» (p. 90) trouve dans la traduction «lorsqu'un procès (...) leur est tout à coup retire» (Goldschmidt, p. 151) une transposition sans équivoque puisque l'élément contextuel 'tout à coup' éclaire le sens du verbe.

Plus nombreux sont par contre les exemples où la traduction diverge du modẻle; ces transpositions auxquelles conduit et peut-être même contraint la nature de la structure passive française sont de plusieurs types; parmi ces derniers, notons la traduction par un verbe 
pronominal. Ce type de transposition rend parfaitement bien le sens passif, mais risque là encore, selon l'aspect lexical du verbe, d'effacer le contenu dynamique de l'auxiliaire 'werden'. Les traductions de «wurde ihm entgegnet» (p. 91) par «il s'entendait répondre» (Goldschmidt, p. 152), de «als (...) die Tür ein wenig geöffnet wurde» (p. 105) par «la porte s'entrebâilla» (Goldschmidt, p. 170) ou encore de «diese Unannehmlichkeit wurde für K. dadurch verstärkt» (p. 109) par "ce désagrément se trouvait encore accentué» (toujours d'après Goldschmidt, p. 176) transposent fidèlement le contenu passif-processus du modèle; par contre la traduction de «Bei einer wirklichen Freisprechung sollen die Prozeßakten vollstăndig abgelegt werden» ( $p$. 116) par «Pour un acquittement réel toutes les pièces du procès doivent se trouver anéanties» (Vialatte, p. 237) risque de biaiser la compréhension de l'auxiliaire 'werden' et de suggérer un passif-état. De plus, si l'usage de la forme pronominale avec connotation passive s'impose avec certains verbes, il paraîtra facilement artificiel et inadéquat dans certains cas, et le dernier exemple en est un témoignage. C'est ce qui explique que cette forme soit relativement peu représentée dans ces trois traductions.

Plus fréquents sont par contre les cas où les traductions transposent les passifs en 'werden' par des formes actives. Ceci représente en gros deux tiers des exemples du chapitre 7. Ces activations sont de deux types.

Dans le premier cas, le processus est présenté sans mention d'agent et ces cas, répétons-le, prédominent; le traducteur restitue alors un agent hypothétique de forme impersonnelle qui devient sujet du verbe à la forme active. Ce type de transposition prévaut dans les trois traductions et tout particulièrement dans la plus ancienne, celle de Vialatte. «[W]erde sie (...) kaum gelesen» (p. 84) devient «on ne la parcourait que de façon fugitive» (Goldschmidt, p. 144); en face de «er wird (...) weitergeleitet» (p. 116), on lit: «on continue à la diriger vers les instances supérieures» (Vialatte, p. 237) ou «le dossier continue à suivre les voies de la procédure» (Lortholary, p. 196).

Cette subjectivation, quoique sous une forme impersonnelle, est cependant absente de la construction allemande et donne à l'action décrite par le verbe un contenu direct et une immédiateté inconnue de 
la forme passive originale. Ce décalage dicté par les contraintes et les propriétés de la forme passive française n'est pourtant pas sans poser certains problèmes. Lorsque le passif est accompagné, comme cela arrive souvent, par une expression de modalité - autre filtre à l'action directe -, le français doit également personnaliser le processus. On lit ainsi:

Die Eingabe mußte gemacht werden. (p. 94)

il fallait établir la requête. (Goldschmidt, p. 156)

Certes, la traduction «il fallait» suggère la dépersonnalisation du processus; cependant, la forme active de l'infinitif opère une transposition de perspective et supprime la dualité processus et finitude de la structure allemande. Dans certains cas, le traducteur va même jusqu'à ajouter le rapport action/sujet: «Gefahren (...), die eben abgewehrt werden mußten» (p. 93) se lit «divers dangers se présentaient, auxquels il lui fallait parer». (Vialatte, p. 198) Avec ou sans mention de la personne impliquée (lui dans le dernier exemple), ces deux demières transpositions supposent pourtant un sujet sousentendu actif. Le point de vue face au procès a totalement changé.

Les exemples de décalage les plus flagrants restent certainement ceux où la traduction opte pour un renversement de perspective: le complément d'agent, s'il existe, devient sujet d'une forme verbale active et le sujet grammatical de la forme passive devient l'objet du verbe, les relations rhématiques et thématiques se trouvant inversées. Ainsi, la question du peintre «Wollen Sie von den Mädchen belastigt werden?» (p. 119) devient «Vous voulez vraiment que les petites filles vous importunent». (Goldschmidt, p. 190) Dans le texte allemand, le sujet actif du premier verbe reste sujet grammatical de la forme passive; il est le centre. Dans le texte français, nous avons changement de sujet et banalisation de la perspective; le narrateur parlant de K. au lecteur dira:

als werde über seinem Kopf von zwei Männern (...) verhandelt (p. 96)

En traduction, cela se lit: 
par-dessus sa tête deux hommes (...) débattaient de son sort. (Lortholary, p. 169)

Cette forme verbale situe le lecteur au cour de l'action et donne tant au personnage principal qu'au lecteur un autre regard, un autre rôle par rapport aux événements du récit. Il n'est pas rare non plus que les traductions ajoutent un intervenant actif absent de l'original:

Immer wieder wurde ein Advokat ausgeschickt (p. 89)

Ils envoyèrent l'un après l'autre des confrères (Lortholary, p. 159);

ou encore:

Verdächtig aber blieben die unaufhörlich hervorgehobenen persőnlichen Beziehungen zu den Beamten. Mußten sie denn ausschließlich zu K.'s Nutzen ausgebeutet werden? (p. 92)

devient

ne les utilisait-il vraiment qu'au profit de K.? (Vialatte, p. 196).

La forme passive allemande laisse délibérément dans l'ombre les acteurs, s'attachant plus au processus; le texte-cible, quant à lui, remplace l'objectivation par l'animisme. Malgré son existence, la forme passive française, élément discursif habituel des règlements et textes de lois, n'a pas, en raison de la polysémie de son auxiliaire, l'emploi qu'elle a en allemand. La multiplicité et la variabilité des équivalences représentées dans les traductions en sont bien la preuve; mais ceci ne suffit peut-être pas à expliquer cette tendance à la subjectivation du traducteur là où l'original aurait pu être respecté: face à «Darin stimmten aber alle überein, daß leichtsinnige Anklagen nicht erhoben werden» (p. 110), on lit dans la traduction de Lortholary «le tribunal ne porte pas d'accusation à la légère» (p. 187), alors que la traduction de Goldschmidt maintient la perspective de l'original en traduisant par «il n'est pas établi d'accusations à la légère». (p. 177) 
Au risque de donner un tour peu familier au texte, cette dernière traduction maintient le plus possible la tournure passive et transmet au texte-cible une composante propre au texte-source. Elle contre ainsi l'animisme et la personnalisation vers lesquels tend plus volontiers, nous semble-t-il, la langue française. Cette tendance, qui s'explique ici par l'absence d'une tournure passive claire, se retrouve à d'autres niveaux discursifs. Pensons ainsi aux usages que fait la langue allemande du pronom impersonnel 'es' avec des verbes exprimant un processus qui, indépendamment de tout référent, se déroule de luimême, ('es tơnt', 'es kracht', 'es läutet'); devant ces formes, la langue française devra ici aussi prefférer la subjectivation ('on entend des bruits, des craquements, des sons de cloches'). En principe, d'ailleurs, n'importe quelle phrase allemande peut prendre cette forme pseudoimpersonnelle, retardant ainsi l'intervention du sujet grammatical; on dira 'es kommt jemand', mais 'quelqu'un vient'. Le français n'use de cette construction qu'avec certains verbes tels que falloir, exister, etc. À cause de structures discursives aussi opposées, Truffault ${ }^{6}$ généralise et constate que «le personnel en français prime le phénoménal, Dieu le numineux et l'ego id. Entre 'es' et 'on', il existe une différence essentielle: l'action sans l'auteur, l'expression absolue de l'action devient en français action avec un auteur inconnu». (pp. 271-272)

Dans les textes de Kafka, les passifs allemands vident le récit du sujet au profit du processus, du procès. La traduction, quant à elle, personnalise, transpose par des verbes pronominaux ou des équivalents sémantiques, enlevant ainsi au texte de Kafka une de ses structures essentielles. Par la diversité et la disparité des transpositions, elle prive le texte de l'effet répétitif des passifs. De par sa polysémie, la forme dite passive en français permet d'autant plus difficilement le passage subtil du passif en 'werden', expression d'une progression et de son effet, au passif en 'sein', expression d'un état, d'une qualité, d'une aptitude d'un sujet. Étant donné l'adéquation des éléments formels qui composent le passif allemand en 'sein' et le passif français en 'être', $50 \%$ des exemples dénombrés dans le chapitre 7 sont traduits littéralement. Mais pour les mêmes raisons que lors de la transposition du passif en 'werden' (contenu inchoatif ou imperfectif du lexème

6. Louis Truffault, Problèmes linguistiques de traduction (Müchen, Max Hueber Verlag, 1983). 
verbal, temps de l'auxiliaire), la transposition littérale risque parfois d'être ambiguè. Ainsi la formulation «auch der Prozess und sogar der Freispruch sind vernichtet, alles ist vernichtet» (p. 116) trouve dans la traduction de Goldschmidt l'équivalent suivant: «mais même le procès et l'acquittement sont anéantis, tout est anéanti». (p. 186) Le verbe 'anéantir', dont l'aspect perfectif ne s'impose pas de prime abord, permet les deux lectures d'un passif. Mais que dire des deux autres traductions qui, par contre, choisissent de souligner l'aspect dynamique du verbe et donnent de ce passage la transposition: "on détruit tout...» (Vialatte, p. 237; Lortholary, p. 196). Quoique l'expression d'un processus soit absente de l'original, c'est la tendance animiste de la langue française qui l'emporte encore. Un quart des exemples de passif en 'sein' se voient transposés de cette façon. L'assimilation des deux passifs va même plus loin encore, puisque le verbe pronominal est investi du rôle de rendre aussi bien l'un que l'autre passif: «Als eine Grundregel für das Verhalten eines Angeklagten erschien es ihm, immer vorbereitet zu sein» (p. 121) devient alors: «la grande règle devait être pour un accusé de se trouver toujours prêt à tout» (Vialatte, p. 244). C'est au lexème adjectival ou verbal que revient l'entière responsabilité de signifier le processus ou l'état.

Comme dans les premiers passifs, l'inversion des rôles et le changement de perspective n'est pas rare non plus; pour "war er (...) vom Prozeß im Grunde wenig betroffen gewesen» (p. 97), on lit «ce procès l'avait au fond peu touché» (Lortholary, p. 170). Bien que ce passif permette plus facilement le calque, on constate que la traduction en français opère les décalages que nous avions déjà observés dans les premiers exemples de passif en 'werden': faute de ne pouvoir rendre le passage du 'werden' au 'sein', la traduction uniformise les deux perspectives des deux passifs, déplace de ce fait les rapports personnage/action et affaiblit l'objet de quête du personnage: cette soumission qui donne une certaine forme de liberté. Nous illustrerons ce phénomène d'uniformisation en comparant les trois traductions d'un passage précédemment cité et dont nous avions souligné la signification:

Sie sind zwar verhaftet [dit la logeuse] aber nicht so wie ein Dieb verhaftet wird. Wenn man wie ein Dieb verhaftet wird, so ist es schlimm. (p. 20) 
Madame Grubach distingue donc bien deux situations, «wie ein Dieb verhaftet werden» et «verhaftet sein» comme K. Dans deux traductions, nous lisons:

vous êtes bien arrêté, mais pas comme on arrête un voleur. Quand on est arrêté comme un voleur, c'est fâcheux (d'après Lortholary, p. 47 et Vialatte, p. 66).

La première forme est une traduction littérale de la formule originale; les deux autres, qui n'en sont qu'une dans l'original, deviennent en transposition successivement une forme active avec sujet impersonnel et un passif. La valeur de processus en est effacée et nous nous retrouvons fâcheusement avec la forme que madame Grubach utilisait lors de son premier constat. Ainsi se trouvent confondus ici le voleur et Josef $\mathrm{K}$ ! La traduction de Goldschmidt se lit comme suit:

vous êtes arrêté, c'est vrai, mais non pas comme est arrêté un voleur; quand on est arrêté comme l'est un voleur, c'est grave. (p. 49)

Ici le traducteur module la seule forme possible du passif français, risquant certes d'estomper les deux points de vue des passifs allemands.

En refusant au traducteur les moyens linguistiques comparables dans leur forme et leur emploi, la langue française devient ici miroir déformant. C'est le défi qu'ont dû relever les trois traductions du Proces. En penchant pour des transpositions propres à la langue française, Vialatte et, dans une certaine mesure, Lortholary ont recherché l'adaptation; en préférant bien souvent à la tournure active, plus naturelle à la langue française, la tournure passive inhabituelle, la traduction de Goldschmidt peut se justifier, car elle tente de rejoindre une couche profonde du texte original: «Telle la tangente qui effleure le cercle en un point, ce seul contact et non le point lui dictant la loi d'après laquelle elle suivra son tracé jusqu'à l'infini, la traduction effleure en ce point infiniment petit le sens de l'original, poursuivant son propre tracé en vertu des lois de la fidélité et de la liberté des 
mouvements de la langue.»

7. Walter Benjamin, «Die Aufgabe des Ubersetzers» (1923), Das Problem des Übersetzens, éd. Hans-Joachim Störig, Wege der Forschung VIII (Darmstadt, 1969, pp. 156-169), p. 167. 